\title{
Kinetics of microRNA Expression in Bronchoalveolar Lavage Fluid Samples
}

\author{
Matthias Brock $^{1} \cdot$ Thomas Rechsteiner $^{1} \cdot$ Malcolm Kohler $^{1} \cdot$ Daniel Franzen $^{1}$ • \\ Lars C. Huber ${ }^{1}$
}

Received: 30 January 2015/Accepted: 16 March 2015/Published online: 21 March 2015

(C) Springer Science+Business Media New York 2015

\begin{abstract}
Levels of microRNAs (miRNAs) are increasingly assessed in biological fluids, for example, in samples obtained by bronchoalveolar lavage (BAL). "Post-collection kinetics" of miRNA expression levels, however, have not been investigated to date. In these experiments, we analyzed the dynamic expression profile of 5 different miRNAs (miR-17, miR-19b, miR-20b, miR-125a, and miR-223-3p) in BAL within the first $24 \mathrm{~h}$ following collection by routine bronchoscopy. miRNAs were quantified $0,1,4,8$, and $24 \mathrm{~h}$ after collection in samples that were kept at $4{ }^{\circ} \mathrm{C}$ or at room temperature. The expression of all five miRNAs was found to remain stable between the first $8 \mathrm{~h}$ after collection. $24 \mathrm{~h}$ after collection miRNAs faced substantial alterations in their expression profile. These data emphasize that BAL samples intended for further miRNA analysis can be handled at room temperature within the first $8 \mathrm{~h}$ after bronchoscopy.
\end{abstract}

Keywords microRNAs - Biofluid samples ·

Bronchoalveolar lavage $\cdot$ Kinetics

\section{Introduction}

microRNAs (miRNAs) are small, non-coding RNA fragments that appear to control the expression of more than $60 \%$ of the human genome [1]. As such, miRNAs have emerged as pathogenetic factors, as potential therapeutic

Lars C. Huber

lars.huber@usz.ch

1 Division of Pulmonology, University Hospital Zurich, Zurich, Switzerland targets and as biomarkers in several pulmonary diseases, including but not limited to pulmonary hypertension, [2-4], obstructive ventilatory disorders [5-7], cystic fibrosis [8], and lung carcinoma [9] (reviewed in [10]). miRNAs show several features that make them attractive as surrogate markers that might be helpful in the diagnostic work-up and prognosis. These features include a high stability, tissue-specific expression and specific and sensitive detection by qPCR technology [11]. The expression of miRNAs has been analyzed in lung tissue and biofluid samples including blood samples and urine and, recently, in bronchoalveolar fluid [12-14]. However, standardized methods that define time points and work-up following collection of miRNAs are lacking and "post-collection" dynamics of miRNA levels in bronchoalveolar fluid have not been addressed so far. Here, we investigated the dynamic expression profile of miRNAs within the first $24 \mathrm{~h}$ following collection by routine bronchoscopy and bronchoalveolar lavage (BAL). The investigated miRNAs included miR-17, miR-19b, and miR-20b, all of which have been described to be expressed in similar levels in serum samples and in samples obtained by BAL [13]. Moreover, the hypoxia-induced miR-125a and the neutrophilic miR-223-3p were analyzed [15].

\section{Methods}

Patients were recruited from 06/2014 to 09/2014 during regular visits in our outpatient clinic. Patient's diagnosis included sarcoidosis (two patients), hypersensitivity pneumonitis (two patients), drug-induced pneumopathy (Pembrolizumab, an anti-programmed-death-receptor-1 antibody used for the treatment of advanced melanoma [16]) and unclear lung disease (one patient each). All patients gave their written informed consent. The study protocol was 
approved by the local ethical committee (approval number KEK-ZH-Nr 2014-0011). Bronchoalveolar fluid samples were obtained during routine bronchoscopy and processed as shown in Fig. 1. Synthetic Caenorhabditis elegans miRNA cel-miR-39 (synthesized by Microsynth, Balgach, Switzerland) were added to $200 \mu \mathrm{l}$ of denatured sample (10 $\mu \mathrm{l}$ of a 5 fmol solution) and used as endogenous spike-in control. miRNAs were isolated using the miRCURY RNA Isolation kit-biofluids (Exiqon, Vedbaek, Denmark). RNA samples containing miRNAs were eluted in $32 \mu \mathrm{l}$ RNase-free water and stored at $-80{ }^{\circ} \mathrm{C}$ for later analysis. Mature miRNA sequences were detected with specific stem-loop primers and reverse transcribed using MultiScribe reverse transcriptase (Life Technologies, Zug, Switzerland) according to Chen et al. [17]. Quantification of complementary DNA (cDNA) was performed by SYBR Green quantitative PCR (qPCR, Applied Biosystem StepOnePlus system, Life Technologies). Sequences of primers used in this study for reverse transcription and amplification of miRNAs were the following: cel-miR-39, RT 5'-GTC GTA TCC AGT GCA GGG TCC GAG GTA TTC GCA CTG GAT ACG ACC AAG CT3', forward 5' - CCT CAC CGG GTG TAA ATC AG-3'; miR17 RT 5'-GTC GTA TCC AGT GCA GGG TCC GAG GTA TTC GCA CTG GAT ACG ACC TAC CT-3', forward 5'GCG GCA AAG TGC TTA CAG TG-3'; miR-19b, RT 5'GTC GTA TCC AGT GCA GGG TCC GAG GTA TTC GCA CTG GAT ACG ACT CAG TT-3', forward 5'-CGG CGG TGT GCA AAT CCA TGC-3'; miR-20b, RT 5'-GTC GTA TCC AGT GCA GGG TCC GAG GTA TTC GCA CTG GAT ACG ACT ACC TG-3', forward 5' - CTC AAA GTG CTC ATA GTG CAG-3'; miR-125a 5'-GTC GTA TCC AGT GCA GGG TCC GAG GTA TTC GCA CTG GAT ACG ACT CAC AG-3', forward 5'-TCC CTG AGA CCC TTT AAC C-3'; miR-223-3p, RT 5'-GTC GTA TCC AGT
GCA GGG TCC GAG GTA TTC GCA CTG GAT ACG ACT GGG GT-3', forward 5'-CCC TGT CAG TTT GTC AAA TAC C- $3^{\prime}$; universal reverse primer $5^{\prime}$-GAG GTA TTC GCA CTG GAT AC- $3^{\prime}$. Obtained signals were normalized to the levels of cel-miR-39. Specific amplification was confirmed by performing melt curve analysis (StepOne software v2.3, Life Technologies). Differential gene expression was calculated with the threshold cycle $\left(\mathrm{C}_{\mathrm{t}}\right)$ method [18].

\section{Results}

Levels of five different miRNAs were assessed at prespecified time points and temperature following collection by bronchoscopy. Since BAL samples were obtained from six patients, the experimental set included 255 independent single-point measurements.

As shown in Fig. 2, expression levels of all five miRNAs were found to be similar between the first $8 \mathrm{~h}$ after collection, independent whether the samples were kept at $4{ }^{\circ} \mathrm{C}$ or at room temperature. Statistical analysis did not reveal any significant changes. Detailed expression levels were as shown in Table 1.

\section{Discussion}

An increasing number of studies has addressed the expression profile of specific miRNAs in bronchoalveolar fluid. Such miRNA signatures might be used as a novel tool of biomarkers for diagnosis, to follow-up patients and to predict outcome. However, while being of major interest, no standardized protocol for obtaining and further processing of miRNAs has been proposed to date. It remained

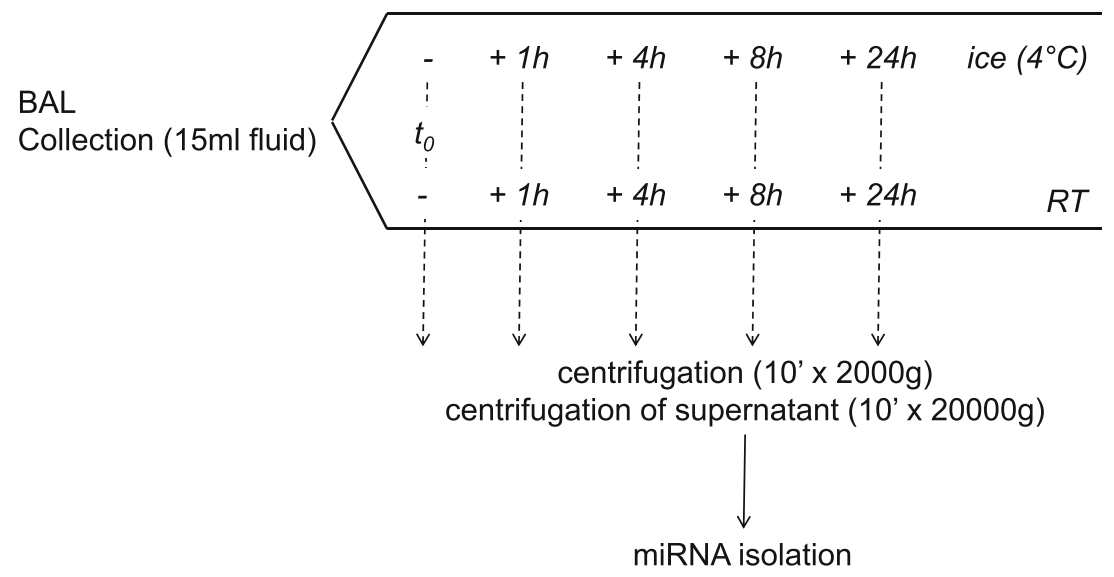

Fig. 1 miRNA isolation from BAL samples. $15 \mathrm{ml}$ of BAL was collected and aliquoted in 8 tubes $(1.5 \mathrm{ml}$ each). Four tubes were kept on ice $\left(4^{\circ} \mathrm{C}\right), 4$ tubes were stored at room temperature. Following defined time points $(0,1,4,8$, and 24 h upon collection), the samples were centrifuged $\left(10^{\prime}\right.$ at $\left.2000 \times g\right)$. Supernatants underwent a second step of centrifugation $\left(10^{\prime}\right.$ at $\left.20,000 \times g\right)$ before isolation of miRNAs. The experimental set included 255 independent analyses 
(a) $4^{\circ} \mathrm{C}$
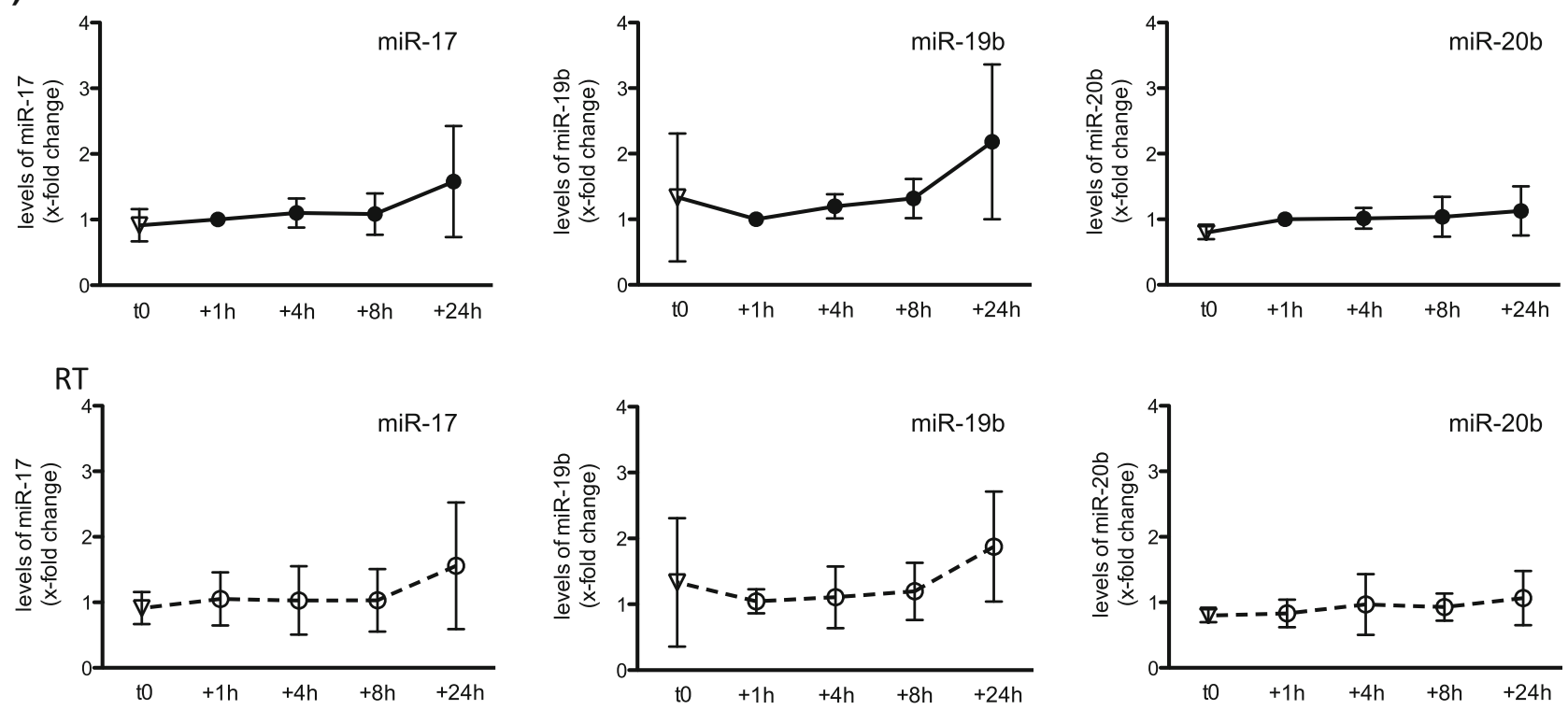

(b) $4^{\circ} \mathrm{C}$
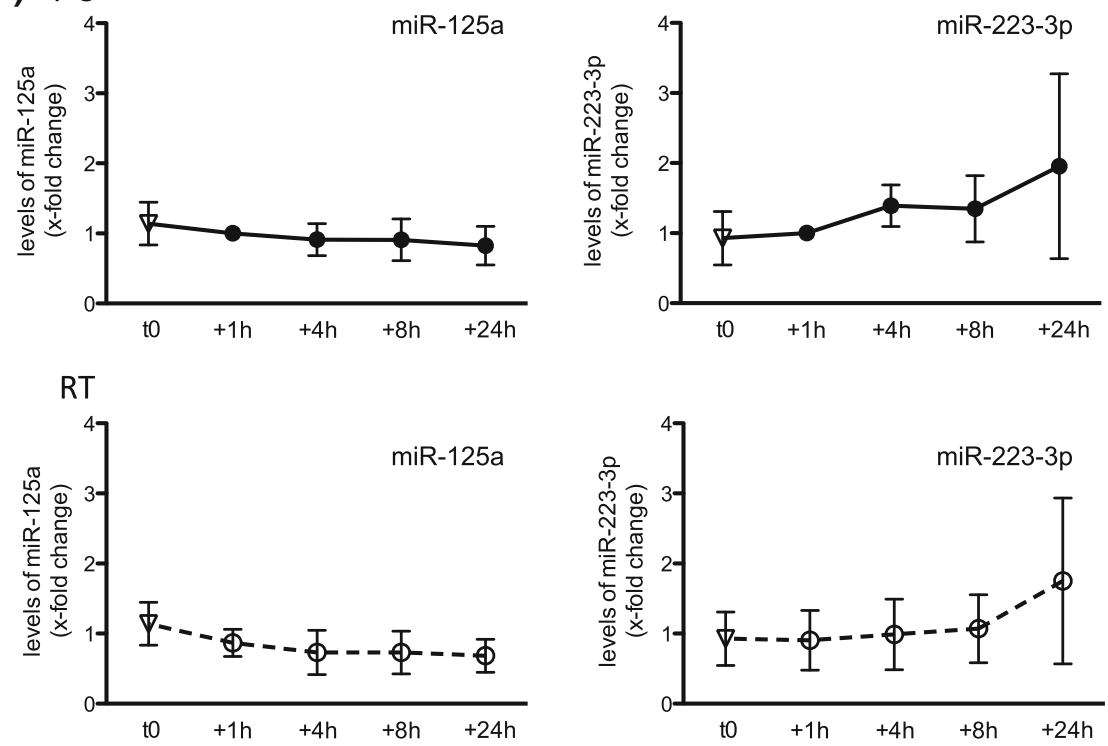

Fig. 2 "Post-collection" kinetics of BAL-derived miRNAs. Expression levels of five different miRNAs were assessed at time point $t_{0}, 1$, 4,8 , and $24 \mathrm{~h}$ after collection both with samples kept at $4{ }^{\circ} \mathrm{C}$ (upper

panel) and at room temperature (lower panel). Expression at time point $1 \mathrm{~h}$ and temperature of $4{ }^{\circ} \mathrm{C}$ was arbitrarily chosen as 1.00 $\left(n=6\right.$, except for $\left.t_{0} n=3\right)$. For details please refer to Table 1

thus unclear whether the expression levels of identified miRNAs are comparable between different study protocols. In this set of experiments, we show that the expression of miRNAs obtained from human BAL samples is stable when assessed at different time points over a period of $8 \mathrm{~h}$. Standard deviations within this time period were not subjected to greater variations highlighting the stability of miRNAs in BAL samples. Conversely, after $24 \mathrm{~h}$, a substantial increase of variations was observed. However, probably due to the wide scattering of individual data points, no significant changes could be detected. In addition, miRNA stability was found to remain unchanged by processing BAL samples on ice or at room temperature. Within these limits, temperature appears to be of minor importance for the miRNA expression levels.

In these experiments, we analyzed the expression levels of miR-17, miR-19b, miR-20b, miR-125a, and miR-2233 p. A role for these miRNAs has already been implicated in the context of diverse pulmonary diseases. miR-17 and miR-19b are members of the important miR-17/92 cluster 
Table 1 miRNA expression profile

\begin{tabular}{|c|c|c|c|c|c|c|c|c|c|}
\hline & \multirow[b]{2}{*}{$t_{0}$} & \multicolumn{4}{|l|}{$4{ }^{\circ} \mathrm{C}$} & \multicolumn{4}{|c|}{ Room temperature } \\
\hline & & $1 \mathrm{~h}$ & $4 \mathrm{~h}$ & $8 \mathrm{~h}$ & $24 \mathrm{~h}$ & $1 \mathrm{~h}$ & $4 \mathrm{~h}$ & $8 \mathrm{~h}$ & $24 \mathrm{~h}$ \\
\hline miR-17 & $0.91 \pm 0.25$ & 1.00 & $1.1 \pm 0.22$ & $1.08 \pm 0.31$ & $1.58 \pm 0.85$ & $1.05 \pm 0.4$ & $1.03 \pm 0.52$ & $1.03 \pm 0.48$ & $1.56 \pm 0.97$ \\
\hline $\operatorname{miR}-19 b$ & $1.33 \pm 0.98$ & 1.00 & $1.2 \pm 0.18$ & $1.32 \pm 0.3$ & $2.18 \pm 1.18$ & $1.05 \pm 0.18$ & $1.11 \pm 0.47$ & $1.2 \pm 0.44$ & $1.88 \pm 0.83$ \\
\hline $\operatorname{miR}-20 b$ & $0.8 \pm 0.1$ & 1.00 & $1.02 \pm 0.16$ & $1.04 \pm 0.3$ & $1.13 \pm 0.37$ & $0.83 \pm 0.21$ & $0.97 \pm 0.46$ & $0.93 \pm 0.21$ & $1.07 \pm 0.41$ \\
\hline miR-125a & $1.14 \pm 0.31$ & 1.00 & $0.91 \pm 0.23$ & $0.91 \pm 0.3$ & $0.83 \pm 0.28$ & $0.87 \pm 0.19$ & $0.73 \pm 0.32$ & $0.73 \pm 0.3$ & $0.68 \pm 0.24$ \\
\hline $\operatorname{miR}-223-3 p$ & $0.93 \pm 0.38$ & 1.00 & $1.39 \pm 0.3$ & $1.35 \pm 0.47$ & $1.96 \pm 1.32$ & $0.91 \pm 0.43$ & $0.99 \pm 0.51$ & $1.07 \pm 0.49$ & $1.75 \pm 1.19$ \\
\hline
\end{tabular}

Expression levels of all five miRNAs obtained and isolated as described in Fig. 1 are shown for all five miRNAs both at $4{ }^{\circ} \mathrm{C}$ and at room temperature. Since samples at time point $t_{0}$ were immediately processed, no distinction between caloric conditions was made

that encodes six different miRNAs modulating, among others, the vascular remodeling of pulmonary arteries [19, 20]. Together with miR-20b, these miRNAs make a panel that can be measured in both the blood and BAL samples [13]. miR-125a is induced by hypoxia and, as such, is of particular interest in pulmonary diseases. miR-223-3p, at least, is known as neutrophilic miRNA and was found to be elevated in lung tissue samples of patients with chronic obstructive disease [5]. These miRNAs, although involved in several pathological processes and being associated with different respiratory disorders, share the property of highly stable post-collection kinetics and feasible detection in BAL samples as shown herein.

Since different miRNAs obtained from six different patients have been assessed at pre-specified conditions, we conclude that the expression levels of miRNAs obtained during BAL are stable over several hours. These data emphasize that BAL samples intended for further miRNA analysis can be handled at room temperature within the first $8 \mathrm{~h}$ after bronchoscopy. Moreover, our results suggest that miRNA data from studies that used different collection protocols (e.g., temperature and time points) are directly comparable when the samples were processed within the first $8 \mathrm{~h}$.

Acknowledgments This study has been supported by the Swiss National Science Foundation (SNF Grant 31003A_144212) and the Olga Mayenfisch Foundation.

Conflict of interest None.

\section{References}

1. Friedman RC, Farh KK, Burge CB et al (2009) Most mammalian mRNAs are conserved targets of microRNAs. Genome Res 19(1):92-105. doi:10.1101/gr.082701.108

2. Brock M, Huber LC (2013) Vascular remodeling in hypoxiainduced pulmonary hypertension: role of cytokines and microRNAs. PVRI Rev 5(1):20-23. doi:10.4103/0974-6013.118829

3. Grant JS, White K, MacLean MR et al (2013) MicroRNAs in pulmonary arterial remodeling. Cell Mol Life Sci 70(23):4479-4494. doi:10.1007/s00018-013-1382-5
4. White KLJ, Chan SY (2012) Holding our breath: the emerging and anticipated roles of microRNA in pulmonary hypertension. Pulm Circ 2(3):278-290

5. Ezzie ME, Crawford M, Cho JH et al (2012) Gene expression networks in COPD: microRNA and mRNA regulation. Thorax 67(2):122-131. doi:10.1136/thoraxjnl-2011-200089

6. Simpson LJ, Patel S, Bhakta NR et al (2014) A microRNA upregulated in asthma airway $\mathrm{T}$ cells promotes $\mathrm{TH} 2$ cytokine production. Nat Immunol 15(12):1162-1170. doi:10.1038/ni.3026

7. Levanen B, Bhakta NR, Torregrosa Paredes P et al (2013) Altered microRNA profiles in bronchoalveolar lavage fluid exosomes in asthmatic patients. J Allergy Clin Immunol 131(3):894-903. doi:10.1016/j.jaci.2012.11.039

8. Weldon S, McNally P, McAuley DF et al (2014) miR-31 dysregulation in cystic fibrosis airways contributes to increased pulmonary cathepsin S production. Am J Respir Crit Care Med 190(2):165-174. doi:10.1164/rccm.201311-1986OC

9. Rodriguez M, Silva J, Lopez-Alfonso A et al (2014) Different exosome cargo from plasma/bronchoalveolar lavage in non-small-cell lung cancer. Genes Chromosom Cancer 53(9):713-724. doi: $10.1002 /$ gec. 22181

10. Rupani H, Sanchez-Elsner T, Howarth P (2013) MicroRNAs and respiratory diseases. Eur Respir J 41(3):695-705. doi:10.1183/ 09031936.00212011

11. Kroh EM, Parkin RK, Mitchell PS et al (2010) Analysis of circulating microRNA biomarkers in plasma and serum using quantitative reverse transcription-PCR (qRT-PCR). Methods 50(4):298-301. doi:10.1016/j.ymeth.2010.01.032

12. Molina-Pinelo S, Pastor MD, Suarez R et al (2014) MicroRNA clusters: dysregulation in lung adenocarcinoma and COPD. Eur Respir J 43(6):1740-1749. doi:10.1183/09031936.00091513

13. Molina-Pinelo S, Suarez R, Pastor MD et al (2012) Association between the miRNA signatures in plasma and bronchoalveolar fluid in respiratory pathologies. Dis Markers 32(4):221-230. doi:10.3233/DMA-2011-0882

14. Brown JN, Brewer HM, Nicora CD et al (2014) Protein and microRNA biomarkers from lavage, urine, and serum in military personnel evaluated for dyspnea. BMC Med Genomics 7:58. doi:10.1186/1755-8794-7-58

15. Johnnidis JB, Harris MH, Wheeler RT et al (2008) Regulation of progenitor cell proliferation and granulocyte function by microRNA223. Nature 451(7182):1125-1129. doi:10.1038/nature06607

16. Robert C, Ribas A, Wolchok JD et al (2014) Anti-programmeddeath-receptor-1 treatment with pembrolizumab in ipilimumabrefractory advanced melanoma: a randomised dose-comparison cohort of a phase 1 trial. Lancet 384(9948):1109-1117. doi:10. 1016/S0140-6736(14)60958-2

17. Chen C, Ridzon DA, Broomer AJ et al (2005) Real-time quantification of microRNAs by stem-loop RT-PCR. Nucleic Acids Res 33(20):e179. doi:10.1093/nar/gni178 
18. Schmittgen TD, Livak KJ (2008) Analyzing real-time PCR data by the comparative $\mathrm{C}(\mathrm{T})$ method. Nat Protoc 3(6):1101-1108. doi:10.1038/nprot.2008.73

19. Brock M, Trenkmann M, Gay RE et al (2009) Interleukin-6 modulates the expression of the bone morphogenic protein receptor type II through a novel STAT3-microRNA cluster 17/92 pathway. Circ Res 104(10):1184-1191. doi:10.1161/CIRCRE SAHA.109.197491
20. Brock M, Samillan VJ, Trenkmann M et al (2014) AntagomiR directed against miR-20a restores functional BMPR2 signalling and prevents vascular remodelling in hypoxia-induced pulmonary hypertension. Eur Heart J 35(45):3203-3211. doi:10.1093/eur heartj/ehs060 